\title{
A New Algebraic Version of Monteiro's Four-Valued Propositional Calculus
}

\author{
Aldo Victorio Figallo1, Estela Bianco², Alicia Ziliani' ${ }^{2}$ \\ ${ }^{1}$ Instituto de Ciencias Básicas, Universidad Nacional de San Juan, San Juan, Argentina \\ ${ }^{2}$ Departamento de Matemática, Universidad Nacional del Sur, Bahía Blanca, Argentina \\ Email: avfigallo@gmail.com, eabiancoenator@gmail.com, aziliani@gmail.com
}

Received 1 May 2014; revised 20 July 2014; accepted 2 August 2014

Copyright (C) 2014 by authors and Scientific Research Publishing Inc.

This work is licensed under the Creative Commons Attribution International License (CC BY).

http://creativecommons.org/licenses/by/4.0/

(c) (i) Open Access

\section{Abstract}

In the XII Latin American Symposium on Mathematical Logic we presented a work introducing a Hilbert-style propositional calculus called four-valued Monteiro propositional calculus. This calculus, denoted by $\mathcal{M}_{4}$, is introduced in terms of the binary connectives $\Rightarrow$ (implication), $\rightarrow$ (weak implication), $\wedge$ (conjunction) and the unary ones $\sim$ (negation) and $\nabla$ (modal operator). In this paper, it is proved that $\mathcal{M}_{4}$ belongs to the class of standard systems of implicative extensional propositional calculi as defined by Rasiowa (1974). Furthermore, we show that the definitions of four-valued modal algebra and $\mathcal{M}_{4}$-algebra are equivalent and, in addition, obtain the completeness theorem for $\mathcal{M}_{4}$. We also introduce the notion of modal distributive lattices with implication and show that these algebras are more convenient than four-valued modal algebras for the study of four-valued Monteiro propositional calculus from an algebraic point of view. This follows from the fact that the implication $\rightarrow$ is one of its basic binary operations.

\section{Keywords}

Mathematical Logic, Hilbert-Style Propositional Calculus, Non-Classical Logics, Four-Valued Monteiro Propositional Calculus

\section{Preliminaries}

In 1978, A. Monteiro introduced four-valued modal algebras as a generalization of 3-valued Łukasiewicz algebras. These algebras raise a genuine interest both from the points of view of algebra and logic, and especially from that of Algebraic Logic. It is worth mentioning that Monteiro expressed his view that in near future these algebras would give rise to a four-valued modal logic with significant applications in computer science. His in- 
sight was essentially right, although we don't know if such applications have yet been developed. On the other hand, it is well known that Font and Rius $(1990,2000)$ have done an exhaustive research into four-valued modal logics. In particular, in (Font \& Rius, 1990) they studied the class of abstract logics projectively generated by the class of logics defined on four-valued modal algebras by the family of their filters and they obtained an axiomatization of them by means of a Gentzen calculus. Furthermore, in (Font \& Rius, 2000) they defined two sentential logics. One of them is algebrizable in the sense of (Blok \& Pigozzi, 1989) and the corresponding algebras are four-valued modal algebras. The other is not algebrizable in the above sense, but its algebraic counterpart is also the class of four-valued modal algebras. These results gave a positive answer to Monteiro's conjecture. Besides, in (Bianco, 2004, 2008) we gave a Hilbert-style propositional calculus, called four-valued Monteiro propositional calculus which we would describe below. An algebraic study of four-valued modal algebras can be found in (Figallo et al., 1991, 1992, 1994, 1995) and (Loureiro, 1980, 1983a, 1983b, 1983c, 1984, 1985). Recall that:

A four-valued modal algebra is an algebra $\langle A, \wedge, \vee, \sim, \nabla, 1\rangle$ of type $(2,2,1,1,0)$ such that the reduct $\langle A, \vee, \wedge, 1\rangle$ is a distributive lattice with greatest element 1 satisfying these conditions:

(L1) $\sim x=x$,

(L2) $\sim(x \wedge y)=\sim x \vee \sim y$,

(L3) $\sim(x \wedge \sim \nabla x)=1$,

(L4) $x \wedge \sim x=\nabla x \wedge \sim x$.

From the definition, it follows that $A$ is a De Morgan algebra (Monteiro, 1960; Birkhoff, 1967). Besides, Loureiro $(1982,1983 a)$ proved que that the variety $\mathcal{T M}$ of these algebras is generated by the algebra $\mathbb{M}_{4}=\left\langle T_{4}, \vee, \wedge, \nabla, \sim, 1\right\rangle$, where

1) $T_{4}=\{0, a, b, 1\}$ is the De Morgan algebra such that $\sim a=a$ and $\sim b=b$, being $a, b$ not comparable;

2) The operation $\nabla$ is defined by $\nabla 0=0$ and $\nabla x=1$ for all $x \neq 0$.

In what follows, we will denote by $\mathfrak{T M}$ the category of four-valued modal algebras and their corresponding homomorphisms.

For the notions of universal algebra including distributive lattices, De Morgan algebras and category theory used in this paper we refer the reader to (Kalman, 1958; Birkhoff, 1967; MacLane, 1971; Burris \& Sankappanavar, 1981).

The structure of the paper is as follows: In Section 1, we develop a Hilbert-style propositional calculus which we call four-valued Monteiro propositional calculus and denote by $\mathcal{M}_{4}$. Besides, we prove that $\mathcal{M}_{4}$ belongs to the class of standard systems of implicative extensional propositional calculi (Rasiowa, 1974). Furthermore, we show that the notions of four-valued modal algebra and $\mathcal{M}_{4}$-algebra are equivalent. Finally, we demonstrate that $\mathcal{M}_{4}$ is consistent and that the completeness theorem holds. From the equivalence established above, we conclude that from any algebra $\langle L, \wedge, \vee \sim, \nabla, 1\rangle \in \mathcal{T M}$ we have that $\langle L, \rightarrow, \Rightarrow \wedge, \sim, \nabla, 1\rangle$ is an $\mathcal{M}_{4}$-algebra where $x \rightarrow y=\nabla \sim x \vee y$ and $x \Rightarrow y=(x \rightarrow y) \wedge(\nabla x \rightarrow \nabla(x \wedge y))$ and conversely, from any $\mathcal{M}_{4}$-algebra we obtain a four-valued modal algebra by defining $x \vee y=\sim(\sim x \wedge \sim y)$. These statements and the fact that $\Rightarrow$ can be defined from $\rightarrow$ allow us to assert that $\mathcal{T M}$ and the variety $\mathcal{M D \mathcal { L } _ { i }}$ generated by

$\mathbb{L}_{4}=\langle\{0, a, b, 1\}, \rightarrow, \wedge, \sim, \nabla, 1\rangle$ are polynomially equivalent. In Section 2 , we introduce the variety $\mathcal{M D \mathcal { L } _ { i }}$ of modal distributive lattices with implication and we show that the category $\mathfrak{M D} \mathfrak{L}_{i}$ of these algebras and their corresponding homomorphisms is equivalent to $\mathfrak{T M}$. Thus, the category theory notably simplify the proof that should be performed to obtain this result by direct calculations. Hence, we determine a new equational description of four-valued modal algebras which is more convenient than the latter to study $\mathcal{M}_{4}$ from an algebraic point of view, since the implication $\rightarrow$ is one of its basic binary operations.

\section{Four-Valued Monteiro Propositional Calculus}

The main aim of this section is to describe the propositional calculus $\mathcal{M}_{4}$ and show that it has four-valued modal algebras as the algebraic counterpart. The terminology and symbols used here coincide in general with those used in (Rasiowa, 1974).

Let $L=\left(A^{0}, F\right)$ be a formalized language of zero order such that in the alphabet $A^{0}=\left(V, L_{0}, L_{1}, L_{2}, U\right)$ the set 
1) $V$ of propositional variables which they will be denoted by $\alpha, \beta, \gamma, \cdots$, is enumerable;

2) $L_{0}$ is empty;

3) $L_{1}$ contains two elements denoted by $\sim$ and $\nabla$ called negation sign and modal operator sign, respectively;

4) $L_{2}$ contains three elements denoted by $\wedge, \rightarrow$ and $\Rightarrow$ called conjunction sign, weak implication sign and implication sign, respectively;

5) $U$ contains two elements denoted by (,) .

Let $F$ be the set of all formulas over $A^{0}$. For any $\alpha, \beta$ in $F$, we shall write for short $\alpha \leftrightarrow \beta$ instead of $(\alpha \Rightarrow \beta) \wedge(\beta \Rightarrow \alpha)$.

We assume that the set $A_{l}$ of logical axioms consists of all formulas of the following form, where $\alpha, \beta, \gamma$ are any formulas in $F$ :

(A1) $\alpha \rightarrow(\beta \rightarrow \alpha)$,

(A2) $(\alpha \rightarrow(\beta \rightarrow \gamma)) \rightarrow((\alpha \rightarrow \beta) \rightarrow(\alpha \rightarrow \gamma))$,

(A3) $(\alpha \wedge \beta) \rightarrow \alpha$,

(A4) $(\alpha \wedge \beta) \rightarrow \beta$,

(A5) $\alpha \rightarrow(\beta \rightarrow(\alpha \wedge \beta))$,

(A6) $\nabla \alpha \rightarrow \nabla(\alpha \wedge \alpha)$,

(A7) $\nabla(\alpha \wedge \beta) \rightarrow \nabla \beta$,

(A8) $(\alpha \rightarrow \beta) \rightarrow(\nabla(\alpha \wedge \beta) \rightarrow(\nabla(\gamma \wedge \alpha) \rightarrow \nabla((\alpha \wedge \gamma) \wedge(\beta \wedge \gamma))))$,

(A9) $\nabla \nabla \alpha \rightarrow \nabla \alpha$,

(A10) $\alpha \rightarrow \nabla \alpha$,

(A11) $\nabla(\alpha \rightarrow \beta) \rightarrow(\alpha \rightarrow \nabla \beta)$,

(A12) $(\alpha \rightarrow \nabla \beta) \rightarrow \nabla(\alpha \rightarrow \beta)$,

(A13) $\nabla(\alpha \rightarrow(\beta \wedge \gamma)) \rightarrow \nabla((\alpha \rightarrow \beta) \wedge(\alpha \rightarrow \gamma))$,

(A14) $(\beta \rightarrow \gamma) \rightarrow((\nabla \alpha \rightarrow \nabla(\alpha \wedge \beta)) \rightarrow((\nabla \beta \rightarrow \nabla(\beta \wedge \gamma)) \rightarrow(\nabla \alpha \rightarrow \nabla(\alpha \wedge \gamma))))$,

(A15) $\nabla((\alpha \wedge \gamma) \wedge(\beta \wedge \gamma)) \rightarrow \nabla((\gamma \wedge \alpha) \wedge(\gamma \wedge \beta))$,

(A16) $(\nabla \alpha \rightarrow \nabla(\alpha \wedge \beta)) \rightarrow(\sim(\alpha \wedge \beta) \rightarrow \sim \alpha)$,

(A17) $\sim \beta \rightarrow \sim(\alpha \wedge \beta)$,

(A18) $(\nabla \alpha \rightarrow \nabla(\alpha \wedge \beta)) \rightarrow((\beta \rightarrow \alpha) \rightarrow(\nabla \sim \alpha \rightarrow \nabla(\sim \alpha \wedge \sim \beta)))$,

(A19) $\nabla \alpha \rightarrow \nabla(\alpha \wedge(\alpha \wedge \sim(\sim \alpha \wedge \sim \beta)))$,

(A20) $\alpha \rightarrow \sim(\sim \alpha \wedge \sim \beta)$,

(A21) $(\alpha \Rightarrow \beta) \leftrightarrow((\alpha \rightarrow \beta) \wedge(\nabla \alpha \rightarrow \nabla(\alpha \wedge \beta)))$,

(A22) $(\alpha \Rightarrow \beta) \leftrightarrow(\alpha \rightarrow \beta)$,

(A23) $\sim(\sim(\gamma \wedge \alpha) \wedge \sim(\beta \wedge \alpha)) \leftrightarrow(\alpha \wedge \sim(\sim \beta \wedge \sim \gamma))$, 
(A24) $\sim(\alpha \wedge \sim \nabla \alpha)$,

(A25) $\sim \alpha \rightarrow(\nabla \alpha \rightarrow \alpha)$,

(A26) $\nabla \alpha \rightarrow \nabla(\alpha \wedge \nabla \alpha)$,

(A27) $\nabla \sim \alpha \rightarrow(\nabla \nabla \alpha \rightarrow \nabla(\sim \alpha \wedge \alpha))$,

(A28) $(\alpha \Rightarrow \beta) \rightarrow((\beta \Rightarrow \alpha) \rightarrow(\nabla(\gamma \Rightarrow \alpha) \rightarrow \nabla((\gamma \Rightarrow \alpha) \wedge(\gamma \Rightarrow \beta))))$,

(A29) $(\alpha \Rightarrow \beta) \rightarrow((\beta \Rightarrow \alpha) \rightarrow(\nabla(\alpha \Rightarrow \gamma) \rightarrow \nabla((\alpha \Rightarrow \gamma) \wedge(\beta \Rightarrow \gamma))))$,

(A30) $\alpha \rightarrow(\nabla \beta \rightarrow \nabla(\beta \wedge \alpha))$.

The consequence operation $C_{\mathcal{L}}$ in $\mathcal{L}=\left(A^{0}, F\right)$ is determined by the set $\mathcal{A}_{l}$ and by the following rule of inference:

(R1) $\frac{\alpha, \alpha \rightarrow \beta}{\beta}$, (Modus Ponens)

The system $\mathcal{M}_{4}=\left(\mathcal{L}, C_{\mathcal{L}}\right)$ thus obtained, will be called four-valued Monteiro propositional calculus. It is worth mentioning that the above connectives are not independent, however, we consider them for simplicity. We shall denote by $\mathcal{T}$ the set of all formulas derivable in $\mathcal{M}_{4}$. As usual, if $\alpha \in \mathcal{T}$ we shall say that $\alpha$ is a theorem of $\mathcal{M}_{4}$ and we shall write $\vdash \alpha$ or $\alpha$.

In Lemma 1.1 we summarize the most important rules and theorems necessary for further development.

Lemma 1.1. In $\mathcal{M}_{4}$ the following rules and theorems hold:

(R2) $\frac{\alpha}{\beta \rightarrow \alpha}$,

(R3) $\frac{\alpha \rightarrow(\beta \rightarrow \gamma)}{(\alpha \rightarrow \beta) \rightarrow(\alpha \rightarrow \gamma)}$,

(T1) $\alpha \rightarrow \alpha$,

(T2) $(\alpha \rightarrow \beta) \rightarrow((\gamma \rightarrow \alpha) \rightarrow(\gamma \rightarrow \beta))$,

(R4) $\frac{\alpha \rightarrow \beta}{(\gamma \rightarrow \alpha) \rightarrow(\gamma \rightarrow \beta)}$,

(R5) $\frac{(\alpha \rightarrow \beta) \rightarrow(\alpha \rightarrow \gamma)}{\beta \rightarrow(\alpha \rightarrow \gamma)}$,

(R6) $\frac{\alpha \rightarrow(\beta \rightarrow \gamma)}{\beta \rightarrow(\alpha \rightarrow \gamma)}$,

(T3) $(\alpha \rightarrow(\alpha \rightarrow \beta)) \rightarrow(\alpha \rightarrow \beta)$,

(T4) $(\alpha \rightarrow \beta) \rightarrow((\beta \rightarrow \gamma) \rightarrow(\alpha \rightarrow \gamma))$,

(R7) $\frac{\alpha \rightarrow \beta, \beta \rightarrow \gamma}{\alpha \rightarrow \gamma}$,

(R8) $\frac{\alpha \rightarrow \beta}{(\beta \rightarrow \gamma) \rightarrow(\alpha \rightarrow \gamma)}$,

(R9) $\frac{\alpha, \beta}{\alpha \wedge \beta}$, 
(R10) $\frac{\alpha, \alpha \Rightarrow \beta}{\beta}$,

(R11) $\frac{\alpha \Rightarrow \beta}{(\alpha \rightarrow \beta) \wedge(\nabla \alpha \rightarrow \nabla(\alpha \wedge \beta))}$,

(R12) $\frac{(\alpha \rightarrow \beta) \wedge(\nabla \alpha \rightarrow \nabla(\alpha \wedge \beta))}{\alpha \Rightarrow \beta}$,

(T5) $\alpha \Rightarrow \alpha$,

(R13) $\frac{\alpha \Rightarrow \beta, \beta \Rightarrow \gamma}{\alpha \Rightarrow \gamma}$,

(R14) $\frac{\alpha}{\beta \Rightarrow \alpha}$,

(T6) $(\alpha \rightarrow \beta) \rightarrow(\nabla(\alpha \wedge \beta) \rightarrow(\nabla(\gamma \wedge \alpha) \rightarrow \nabla(\beta \wedge \gamma)))$,

(T7) $\nabla(\alpha \wedge \beta) \rightarrow \nabla(\beta \wedge \alpha)$,

(T8) $\nabla(\alpha \wedge \beta) \rightarrow \nabla \alpha$,

(T9) $(\gamma \rightarrow \alpha) \rightarrow((\gamma \rightarrow \beta) \rightarrow(\gamma \rightarrow(\alpha \wedge \beta)))$,

(T10) $\nabla(\alpha \wedge \beta) \rightarrow(\nabla \alpha \wedge \nabla \beta)$,

(T11) $\nabla(\alpha \wedge \beta) \rightarrow \nabla(\nabla \alpha \wedge \nabla \beta)$,

(R15) $\frac{\alpha \Rightarrow \beta}{\nabla \alpha \Rightarrow \nabla \beta}$,

(T12) $(\alpha \wedge \beta) \rightarrow(\beta \wedge \alpha)$,

(T13) $(\sim(\alpha \wedge \beta) \rightarrow \sim \alpha) \rightarrow(\sim \beta \rightarrow \sim \alpha)$,

(R16) $\frac{\alpha \Rightarrow \beta, \beta \Rightarrow \alpha}{\sim \alpha \Rightarrow \sim \beta}$,

(T13) $(\beta \rightarrow \alpha) \rightarrow((\alpha \rightarrow \nabla \gamma) \rightarrow(\beta \rightarrow \nabla \gamma))$,

(R17) $\frac{\beta \Rightarrow \alpha, \gamma \Rightarrow \delta}{(\alpha \rightarrow \gamma) \Rightarrow(\beta \rightarrow \delta)}$,

(R18) $\frac{\alpha \Rightarrow \beta, \gamma \Rightarrow \delta}{(\alpha \wedge \gamma) \Rightarrow(\beta \wedge \delta)}$,

(R19) $\frac{\alpha \Rightarrow \beta, \beta \Rightarrow \alpha, \gamma \Rightarrow \delta, \delta \Rightarrow \gamma}{(\alpha \Rightarrow \gamma) \Rightarrow(\beta \Rightarrow \delta)}$.

Proof. We only prove (R15), (R16) and (R17).

(R15):

1) $\alpha \Rightarrow \beta$,

2) $\alpha \rightarrow \beta$, [(1), (R11), (A3), (r1)]

3) $\nabla \alpha \rightarrow \nabla(\alpha \wedge \beta)$, [(1), (R11), (A4), (r1)] 

4) $(\nabla \alpha \rightarrow \nabla(\alpha \wedge \beta)) \rightarrow(\nabla \alpha \rightarrow \nabla \beta)$, [(A7), (R4)]
5) $\nabla \alpha \rightarrow \nabla \beta$, [(3), (4), (r1)]
6) $(\nabla \alpha \rightarrow(\nabla \alpha \wedge \beta)) \rightarrow(\nabla \alpha \rightarrow \nabla(\nabla \alpha \wedge \nabla \beta))$, [(T11), (R4)]
7) $\nabla \alpha \rightarrow \nabla(\nabla \alpha \wedge \nabla \beta)$, [(3), (6), (r1)]
8) $\nabla \nabla \alpha \rightarrow \nabla(\nabla \alpha \wedge \nabla \beta)$, [(A9), (7), (R7)]
9) $\nabla \alpha \Rightarrow \nabla \beta$. [(5), (8), (R9), (R12)]

\section{(R16):}

1) $\alpha \Rightarrow \beta$,

2) $\beta \Rightarrow \alpha$,

3) $\nabla \alpha \rightarrow \nabla(\alpha \wedge \beta)$, [(1), (R11), (A4)]

4) $\beta \rightarrow \alpha,[(2)$, (R11), (A3)]

5) $\nabla \beta \rightarrow \nabla(\beta \wedge \alpha)$, [(2), (R11), (A4)]

6) $\sim(\beta \wedge \alpha) \rightarrow \sim \beta$, [(5), (A16), (r1)]

7) $\sim \alpha \rightarrow \sim \beta$, [(6), (T13), (r1)]

8) $\nabla \sim \alpha \rightarrow \nabla(\sim \alpha \wedge \sim \beta)$, [(A18), (3), (4), (r1)]

9) $\sim \alpha \Rightarrow \sim \beta$. [(7), (8), (R9), (R12)]

\section{(R17):}

1) $\beta \Rightarrow \alpha$,

2) $\gamma \Rightarrow \delta$,

3) $\beta \rightarrow \alpha$, [(1), (R11), (A3)]

4) $\gamma \rightarrow \delta$, [(2), (R11), (A3)]

5) $\nabla \gamma \rightarrow \nabla(\gamma \wedge \delta)$, [(2), (R11), (A4)]

6) $(\alpha \rightarrow \gamma) \rightarrow(\beta \rightarrow \gamma)$, [(T4), (3), (r1)]

7) $(\alpha \rightarrow \nabla \gamma) \rightarrow(\beta \rightarrow \nabla \gamma)$, [(T14), (3), (r1)]

8) $(\nabla(\alpha \rightarrow \gamma) \rightarrow \nabla(\alpha \rightarrow \gamma)) \rightarrow(\nabla(\alpha \rightarrow \gamma) \rightarrow(\nabla(\beta \rightarrow \gamma)) \rightarrow(\nabla(\alpha \rightarrow \gamma) \rightarrow \nabla((\alpha \rightarrow \gamma) \wedge(\beta \rightarrow \gamma)))$, [(T9)]

9) $(\nabla(\alpha \rightarrow \gamma) \rightarrow(\nabla(\beta \rightarrow \gamma))) \rightarrow(\nabla(\alpha \rightarrow \gamma) \rightarrow \nabla((\alpha \rightarrow \gamma) \wedge(\beta \rightarrow \gamma)))$, [(8), (T1), (r1)]

10) $(\alpha \rightarrow \nabla \gamma) \rightarrow \nabla(\beta \rightarrow \gamma)$, [(7), (A12), (R7)]

11) $\nabla(\alpha \rightarrow \gamma) \rightarrow \nabla(\beta \rightarrow \gamma)$, [(10), (A11), (R7)]

12) $\nabla(\alpha \rightarrow \gamma) \rightarrow(\nabla((\alpha \rightarrow \gamma) \wedge(\beta \rightarrow \gamma)))$, [(11), (9), (r1)]

13) $(\alpha \rightarrow \gamma) \Rightarrow(\beta \rightarrow \gamma)$, [(6), (12), (R9), (R12)]

14) $(\beta \rightarrow \gamma) \rightarrow(\beta \rightarrow \delta)$, [(T2), (4), (r1)] 
15) $(\beta \rightarrow \nabla \gamma) \rightarrow(\beta \rightarrow \nabla(\gamma \wedge \delta))$, [(5), (R4)]

16) $\nabla(\beta \rightarrow \gamma) \rightarrow(\beta \rightarrow \nabla(\gamma \wedge \delta))$, [(A11), (15), (R7)]

17) $(\beta \rightarrow \nabla(\gamma \wedge \delta)) \rightarrow \nabla(\beta \rightarrow(\gamma \wedge \delta))$, [(A12)]

18) $\nabla(\beta \rightarrow \gamma) \rightarrow \nabla(\beta \rightarrow(\gamma \wedge \delta))$, [(16), (17), (R7)]

19) $\nabla(\beta \rightarrow \gamma) \rightarrow \nabla((\beta \rightarrow \gamma) \wedge(\beta \rightarrow \delta))$, [(18), (A13), (R7)]

20) $(\beta \rightarrow \gamma) \Rightarrow(\beta \rightarrow \delta)$, [(14), (19), (R9), (R12)]

21) $(\alpha \rightarrow \gamma) \Rightarrow(\beta \rightarrow \delta)$. [(13), (20), (R13)] ם

Theorem 1.1. The propositional calculus $\mathcal{M}_{4}$ belongs to the class of standard systems of implicative extensional propositional calculi.

Proof. We have to prove that conditions (s1) to (s8) in (Rasiowa, 1974) are satisfied. Clearly, (s1) and (s2) are verified. Besides, (s3), (s4), (s5) and (s6) follow from (T5), (R10), (R13) and (R14), respectively. On the other hand, taking into account (R15) and (R16), we have that (s7) holds. Finally, (R17), (R18) and (R19) allow us to conclude (s8).

Next, our attention is focused on stating the relationship between four-valued modal algebras and $\mathcal{M}_{4}$ algebras (Rasiowa, 1974). Lemma 1.2 will be fundamental for this purpose.

Lemma 1.2. In $\mathcal{M}_{4}$ the following theorems hold:

(T15) $((\alpha \rightarrow \beta) \rightarrow(\alpha \rightarrow \gamma)) \rightarrow(\beta \rightarrow(\alpha \rightarrow \gamma))$,

(T16) $(\alpha \rightarrow(\beta \rightarrow \gamma)) \rightarrow(\beta \rightarrow(\alpha \rightarrow \gamma))$,

(T17) $(\alpha \rightarrow(\beta \rightarrow \gamma)) \rightarrow((\alpha \wedge \beta) \rightarrow \gamma)$,

(T18) $((\alpha \rightarrow \beta) \wedge(\alpha \rightarrow \gamma)) \rightarrow(\alpha \rightarrow(\beta \wedge \gamma))$,

(T19) $\alpha \rightarrow(\alpha \wedge \sim(\sim \alpha \wedge \sim \beta))$,

(T20) $\nabla(\alpha \wedge \sim(\sim \alpha \wedge \sim \beta)) \rightarrow \nabla((\alpha \wedge \sim(\sim \alpha \wedge \sim \beta)) \wedge \alpha)$.

(T21) $(\alpha \rightarrow \beta) \rightarrow(\nabla(\alpha \wedge \beta) \rightarrow(\nabla(\gamma \wedge \alpha) \rightarrow \nabla((\gamma \wedge \alpha) \wedge(\gamma \wedge \beta))))$,

(T22) $(\sim \alpha \wedge \alpha) \rightarrow(\sim \alpha \wedge \nabla \alpha)$,

(T23) $(\sim \alpha \wedge \nabla \alpha) \rightarrow(\sim \alpha \wedge \alpha)$,

(T24) $\nabla(\sim \alpha \wedge \alpha) \rightarrow \nabla(\alpha \wedge \nabla \alpha)$,

(T25) $\nabla(\sim \alpha \wedge \alpha) \rightarrow \nabla((\alpha \wedge \nabla \alpha) \wedge(\sim \alpha \wedge \nabla \alpha))$,

(T26) $\nabla(\sim \alpha \wedge \nabla \alpha) \rightarrow \nabla(\sim \alpha \wedge \alpha)$,

(T27) $\nabla(\sim \alpha \wedge \nabla \alpha) \rightarrow \nabla((\sim \alpha \wedge \nabla \alpha) \wedge(\sim \alpha \wedge \alpha))$.

Proof. We only prove (T17), (T20), (T22) and (T27).

(T17):

1) $(\alpha \wedge \beta) \rightarrow \beta$, [(A4)]

2) $(\beta \rightarrow \gamma) \rightarrow((\alpha \wedge \beta) \rightarrow \gamma)$, [(1), (R8)] 
3) $(\alpha \rightarrow(\beta \rightarrow \gamma)) \rightarrow(\alpha \rightarrow((\alpha \wedge \beta) \rightarrow \gamma))$, [(2), (R4)]

4) $(\alpha \rightarrow((\alpha \wedge \beta) \rightarrow \gamma)) \rightarrow((\alpha \wedge \beta) \rightarrow(\alpha \rightarrow \gamma))$, [(T16)]

5) $((\alpha \rightarrow(\beta \rightarrow \gamma)) \rightarrow(\alpha \rightarrow((\alpha \wedge \beta) \rightarrow \gamma))) \rightarrow((\alpha \rightarrow(\beta \rightarrow \gamma)) \rightarrow((\alpha \wedge \beta) \rightarrow(\alpha \rightarrow \gamma)))$, [(4), (R4)]

6) $(\alpha \rightarrow(\beta \rightarrow \gamma)) \rightarrow((\alpha \wedge \beta) \rightarrow(\alpha \rightarrow \gamma))$, [(3), (5), (r1)]

7) $((\alpha \wedge \beta) \rightarrow(\alpha \rightarrow \gamma)) \rightarrow(((\alpha \wedge \beta) \rightarrow \alpha) \rightarrow((\alpha \wedge \beta) \rightarrow \gamma))$, [(A2)]

8) $((\alpha \wedge \beta) \rightarrow \alpha) \rightarrow(((\alpha \wedge \beta) \rightarrow(\alpha \rightarrow \gamma)) \rightarrow((\alpha \wedge \beta) \rightarrow \gamma))$, [(7), (R6)]

9) $((\alpha \wedge \beta) \rightarrow(\alpha \rightarrow \gamma)) \rightarrow((\alpha \wedge \beta) \rightarrow \gamma)$, [(A3), (8), (r1)]

10) $((\alpha \rightarrow(\beta \rightarrow \gamma)) \rightarrow((\alpha \wedge \beta) \rightarrow(\alpha \rightarrow \gamma))) \rightarrow((\alpha \rightarrow(\beta \rightarrow \gamma)) \rightarrow((\alpha \wedge \beta) \rightarrow \gamma))$, [(9), (R4)]

11) $(\alpha \rightarrow(\beta \rightarrow \gamma)) \rightarrow((\alpha \wedge \beta) \rightarrow \gamma)$. [(6), (10), (r1)]

\section{(T20):}

1) $\nabla \alpha \rightarrow \nabla(\alpha \wedge(\alpha \wedge \sim(\sim \alpha \wedge \sim \beta)))$, [(A19)]

2) $(\nabla(\alpha \wedge \sim(\sim \alpha \wedge \sim \beta)) \rightarrow \nabla \alpha) \rightarrow(\nabla(\alpha \wedge \sim(\sim \alpha \wedge \sim \beta)) \rightarrow \nabla(\alpha \wedge(\alpha \wedge \sim(\sim \alpha \wedge \sim \beta))))$, [(1), (R4)]

3) $\nabla(\alpha \wedge \sim(\sim \alpha \wedge \sim \beta)) \rightarrow \nabla(\alpha \wedge(\alpha \wedge \sim(\sim \alpha \wedge \sim \beta)))$, [(2), (T8), (r1)]

4) $\nabla(\alpha \wedge(\alpha \wedge \sim(\sim \alpha \wedge \sim \beta))) \rightarrow \nabla((\alpha \wedge \sim(\sim \alpha \wedge \sim \beta)) \wedge \alpha)$, [(T7)]

5) $\nabla(\alpha \wedge \sim(\sim \alpha \wedge \sim \beta)) \rightarrow \nabla((\alpha \wedge \sim(\sim \alpha \wedge \sim \beta)) \wedge \alpha)$. [(3), (4), (R7)]

(T22):

1) $(((\sim \alpha \wedge \alpha) \rightarrow \sim \alpha) \wedge((\sim \alpha \wedge \alpha) \rightarrow \nabla \alpha)) \rightarrow((\sim \alpha \wedge \alpha) \rightarrow(\sim \alpha \wedge \nabla \alpha))$, [(T18)]

3) $(\sim \alpha \wedge \alpha) \rightarrow \sim \alpha$, [(A3)]

4) $(\sim \alpha \rightarrow(\sim \alpha \wedge \nabla \alpha)) \rightarrow((\sim \alpha \wedge \alpha) \rightarrow \nabla \alpha)$, [(T17)]

5) $\alpha \rightarrow \nabla \alpha,[(\mathrm{A} 10)]$

6) $\sim \alpha \rightarrow(\alpha \wedge \nabla \alpha)$, [(4), (R2)]

7) $(\sim \alpha \wedge \alpha) \rightarrow \nabla \alpha$, [(3), (5), (r1)]

8) $((\sim \alpha \wedge \alpha) \rightarrow \sim \alpha) \wedge((\sim \alpha \wedge \alpha) \rightarrow \nabla \alpha)$, [(2), (6), (R9)]

9) $(\sim \alpha \wedge \alpha) \rightarrow(\sim \alpha \wedge \nabla \alpha)$. [(7), (1), (r1)]

(T27):

1) $\nabla(\sim \alpha \wedge \nabla \alpha) \rightarrow \nabla(\sim \alpha \wedge \alpha)$, [(T26)]

2) $(\nabla(\sim \alpha \wedge \alpha) \rightarrow \nabla((\sim \alpha \wedge \nabla \alpha) \wedge(\sim \alpha \wedge \alpha))) \rightarrow(\nabla(\sim \alpha \wedge \nabla \alpha) \rightarrow \nabla((\sim \alpha \wedge \nabla \alpha) \wedge(\sim \alpha \wedge \alpha)))$, [(1), (R8)]

3) $\nabla((\sim \alpha \wedge \alpha) \wedge(\sim \alpha \wedge \nabla \alpha)) \rightarrow \nabla((\sim \alpha \wedge \nabla \alpha) \wedge(\sim \alpha \wedge \alpha))$, [(T7)]

4) $(\nabla(\sim \alpha \wedge \alpha) \rightarrow \nabla((\sim \alpha \wedge \alpha) \wedge(\sim \alpha \wedge \nabla \alpha))) \rightarrow(\nabla(\sim \alpha \wedge \alpha) \rightarrow \nabla((\sim \alpha \wedge \nabla \alpha) \wedge(\sim \alpha \wedge \alpha)))$, [(3), (R4)] 
5) $\nabla(\sim \alpha \wedge \alpha) \rightarrow \nabla((\sim \alpha \wedge \nabla \alpha) \wedge(\sim \alpha \wedge \alpha))$, [(4), (T25), (r1)]

6) $\nabla(\sim \alpha \wedge \nabla \alpha) \rightarrow \nabla((\sim \alpha \wedge \nabla \alpha) \wedge(\sim \alpha \wedge \alpha))$. [(5), (2), (r1)] $\square$

Remark 1.1. It is worth noting that if $\alpha$ is a formula derivable in $\mathcal{M}_{4}$, then $\alpha_{\mathcal{U}}(v)=1$ for every valuation $v$ of $\mathcal{L}$ in every $\mathcal{M}_{4}$-algebra $\mathcal{U}$.

Proposition 1.1. Let $\langle L, \wedge, \vee, \sim, \nabla, 1\rangle \in \mathcal{T M}$. Then $\langle L, \Rightarrow, \rightarrow, \wedge, \sim, \nabla, 1\rangle$ is an $\mathcal{M}_{4}$-algebra, where $\rightarrow$ and $\Rightarrow$ are defined as follows:

$$
\begin{gathered}
x \rightarrow y=\nabla \sim x \vee y, \\
x \Rightarrow y=(x \rightarrow y) \wedge(\nabla x \rightarrow \nabla(x \wedge y)) .
\end{gathered}
$$

Proof. We shall prove that conditions (a1) to (a4) in (Rasiowa, 1974) hold. Indeed, taking into account the definitions of $\rightarrow$ and $\Rightarrow$ we have that (a1) and (a2) are satisfied. On the other hand, let $a, b \in L$ be such that $a \Rightarrow b=b \Rightarrow c=1$. Then, we get (1) $a \rightarrow b=b \rightarrow c=1$ and (2) $\nabla a \rightarrow \nabla(a \wedge b)=\nabla b \rightarrow \nabla(b \wedge c)=1$. From (1), (A2) and (2), (A14) we have that $a \rightarrow c=1$ and $\nabla a \rightarrow \nabla(a \wedge c)=1$ respectively. These assertions and the fact that $x \leq y$ if and only if $x \rightarrow y=1$ and $\nabla x \rightarrow \nabla(x \wedge y)=1$ allow us to conclude that $a=c$ and so, (a3) holds. Besides, if $a \Rightarrow b=b \Rightarrow a=1$, then by an analogous argument to that used in the proof of (a3), we get $a=b$, from which we infer (a4). $\square$

Proposition 1.2. Let $\langle L, \Rightarrow, \rightarrow, \wedge, \sim, \nabla, 1\rangle$ be an $\mathcal{M}_{4}$-algebra. Then $\langle L, \wedge, \vee, \sim, \nabla, 1\rangle \in \mathcal{T M}$ where $a \vee b=\sim(\sim a \wedge \sim b)$.

Proof. To show that $\langle L, \wedge, \vee, \sim, 1\rangle$ is a De Morgan algebra, by (Marona, 1964) it suffices to prove that conditions (M1) $a=a \wedge \sim(\sim a \wedge \sim b)$ and (M2) $a \wedge \sim(\sim b \wedge \sim c)=\sim(\sim(c \wedge a) \wedge \sim(b \wedge a))$ are satisfied. By virtue of Remark 1.1 and (a4) in (Rasiowa, 1974), (M2) follows from (A23) and (M1) from (A3), (A19), (T19), (T20), (R9) and (R12). Moreover, following an analogous reasoning and taking into account (A24) we infer (L3); besides, (T22), (T23), (T24), (T27), (R9) and (R12) allow us to conclude (L4). Hence, the proof is complete. $\square$

From Propositions 1.1 and 1.2 we infer:

Theorem 1.2. The notions of $\mathcal{M}_{4}$-algebra and four-valued modal algebra are equivalent.

Let $\equiv$ be the binary relation on $F$ defined as follows:

$\alpha \equiv \beta$ if and only if $\vdash \alpha \Rightarrow \beta$ and $\vdash \beta \Rightarrow \alpha$ in $\mathcal{M}_{4}$.

Then, $\equiv$ is a congruence relation on $\langle F, \Rightarrow, \rightarrow, \wedge, \sim, \nabla\rangle$ and $\mathcal{T}$ determines an equivalence class which will be denoted by 1 . Moreover, $\langle F / \equiv, \Rightarrow, \rightarrow, \wedge, \sim, \nabla, 1\rangle$ is an $\mathcal{M}_{4}$-algebra (Rasiowa, 1974) and therefore from Proposition 1.2, we conclude:

Theorem 1.3. $F=\langle F / \equiv, \wedge, \sim, \nabla, 1\rangle \in \mathcal{T} \mathcal{M}$.

On the other hand, since $\mathcal{M}_{4}$ is consistent, from (Rasiowa, 1974) and Theorem 1.2 we have that the completeness theorem for $\mathcal{M}_{4}$ holds, which is included in.

Theorem 1.4. Let $\alpha$ be a formula of $\mathcal{M}_{4}$. Then the following conditions are equivalent:

1) $\alpha$ is derivable in $\mathcal{M}_{4}$;

2) $\alpha$ is valid in every $\mathcal{M}_{4}$-algebra;

3) $\alpha_{\mathcal{F}}\left(v^{0}\right)=1$, where $v^{0}$ is the canonical valuation in the algebra $\mathcal{F}$.

\section{Categorical Equivalence between $\mathfrak{T M}$ and $\mathfrak{M D} \mathfrak{L}_{i}$}

In this section we introduce the notion of modal distributive lattice with implication and we prove some properties of these algebras which allow us to show the announced categorical equivalence.

Definition 2.1. A modal distributive lattice with implication (or $\mathrm{mdl}_{i}$-algebra) is an algebra

$\langle A, \wedge, \vee, \rightarrow, \nabla, 0,1\rangle$ of type $(2,2,2,1,0,0)$ where $\langle A, \wedge, \vee, 0,1\rangle$ is a bounded disitributive lattice satisfying the following identities:

(I1) $x \rightarrow x=1$,

(I2) $x \rightarrow(y \rightarrow z)=(x \rightarrow y) \rightarrow(x \rightarrow z)$,

(I3) $(x \rightarrow y) \rightarrow x=x$, 
(I4) $(x \wedge y) \rightarrow z=x \rightarrow(y \rightarrow z)$,

(I5) $x \rightarrow(y \wedge z)=(x \rightarrow y) \wedge(x \rightarrow z)$,

(I6) $(x \rightarrow y) \wedge y=y$,

(I7) $\nabla 0=0$,

(I8) $\nabla(\nabla x \wedge y)=\nabla x \wedge \nabla y$,

(I9) $\nabla(x \rightarrow y)=x \rightarrow \nabla y$,

(I10) $x \vee(x \rightarrow y)=1$,

(I11) $(\nabla x \rightarrow \nabla(x \rightarrow y)) \rightarrow \nabla((\nabla x \rightarrow x) \wedge(\nabla y \rightarrow y))=1$,

(I12) $(\nabla x \rightarrow \nabla(x \rightarrow y)) \rightarrow x=(\nabla x \rightarrow \nabla(x \wedge y)) \rightarrow(x \wedge((x \rightarrow y) \rightarrow y))$.

In Proposition 2.1 we show some properties of $m \mathrm{ml}_{i}$-algebras which will be useful in what follows.

Proposition 2.1. Let $A$ be an $\mathrm{mdl}_{i}$-algebra. Then the following identities hold:

(I13) $1 \rightarrow x=x$,

(I14) $x \rightarrow 1=1$,

(I15) $\nabla(\nabla x \rightarrow x)=1$,

(I16) $\nabla 1=1$,

(I17) $x \rightarrow \nabla x=1$,

(I18) $\nabla x \rightarrow \nabla(x \wedge \nabla x)=1$,

(I19) $x \rightarrow(x \wedge y)=x \rightarrow y$,

(I20) $x \rightarrow(y \rightarrow x)=1$,

(I21) $x \rightarrow(y \rightarrow z)=y \rightarrow(x \rightarrow z)$,

(I22) $(\nabla x \rightarrow \nabla(x \wedge y)) \rightarrow x=(x \rightarrow y) \rightarrow((\nabla x \rightarrow \nabla(x \wedge y)) \rightarrow(x \wedge y))$,

(I23) $x \wedge \nabla x=x$,

(I24) $x \leq y$ implies $\nabla x \leq \nabla y$,

(I25) $x \leq y$ implies $z \rightarrow x \leq z \rightarrow y$,

(I26) $x \leq y$ if and only if $x \rightarrow y=1, \nabla x \rightarrow \nabla(x \wedge y)=1$,

(I27) $x \leq y$ implies $(y \rightarrow z) \rightarrow(x \rightarrow z)=1$,

(I28) $((\nabla x \rightarrow x) \rightarrow x) \rightarrow \nabla x=1$,

(I29) $\nabla \nabla x=\nabla x$.

Proof. We only prove (I22) and (I26).

(I22): From (I21), (I5), (I3) and (I12) we have that

$$
\begin{aligned}
(x \rightarrow y) \rightarrow((\nabla x \rightarrow \nabla(x \wedge y)) \rightarrow(x \wedge y)) & =(\nabla x \rightarrow \nabla(x \wedge y)) \rightarrow((x \rightarrow y) \rightarrow(x \wedge y)) \\
& =((\nabla x \rightarrow \nabla(x \wedge y)) \rightarrow(x \wedge((x \rightarrow y) \rightarrow y)) . \\
& =(\nabla x \rightarrow \nabla(x \wedge y)) \rightarrow x
\end{aligned}
$$


(I26): Let $x \leq y$. Then, from (I4), (I1), and (I14) we conclude that $x \rightarrow y=1$. Besides, (I1) allows us to infer $\nabla x \rightarrow \nabla(x \wedge y)=1$. Conversely, from the hypothesis, (I13) and (I12) we have that $x=(\nabla x \rightarrow \nabla(x \wedge y)) \rightarrow x=(\nabla x \rightarrow \nabla(x \wedge y)) \rightarrow(x \wedge((x \wedge y) \rightarrow y))=x \wedge y$, and so, $x \leq y . \square$

Let $\mathcal{A}=\langle A, \wedge, \vee, \rightarrow, \nabla, 0,1\rangle$ be an $m d_{l}$-algebra. Then, we define $\Theta(\mathcal{A})=\langle A, \wedge, \vee, \sim, \nabla, 0,1\rangle$, where $\sim x=(x \rightarrow 0) \wedge(\nabla x \rightarrow x)$ for every $x \in A$. Furthermore, given the $m d_{i}$-algebras $\mathcal{A}, \mathcal{A}^{\prime}$ and a homomorphism $f: \mathcal{A} \rightarrow \mathcal{A}^{\prime}$, we define $\Theta(f): \Theta(\mathcal{A}) \rightarrow \Theta\left(\mathcal{A}^{\prime}\right)$ by $\Theta(f)=f$.

Lemma 2.1 and Propositions 2.2, 2.3 and 2.4 are fundamental in order to prove Theorem 2.1.

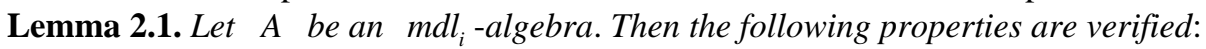

(I30) $\nabla x \wedge(\nabla x \rightarrow x)=x$,

(I31) $(\nabla x \rightarrow x) \rightarrow x=\nabla x$,

(I32) $((x \rightarrow 0) \rightarrow 0) \rightarrow x=1$,

(I33) $\nabla(x \rightarrow 0)=x \rightarrow 0$,

(I34) $((x \rightarrow 0) \wedge(\nabla x \rightarrow x)) \rightarrow 0=\nabla x$,

(I35) $\nabla \sim x=x \rightarrow 0$,

(I36) $x \leq y$ implies $(y \rightarrow 0) \rightarrow(x \rightarrow 0)=1$,

(I37) $x \leq y$ implies $(\nabla y \rightarrow y) \rightarrow(\nabla x \rightarrow x)=\nabla x \rightarrow(y \rightarrow x)$,

(I38) $\nabla(\sim y \wedge \sim x)=(y \rightarrow 0) \wedge(x \rightarrow 0)$,

(I39) $x \leq y$ implies $\sim y \leq \sim x$.

Proof. We only prove (I30) and (I39).

(I30): From (I5), (I17), (I20) and (I4) we have that $x \rightarrow(\nabla x \wedge(\nabla x \rightarrow x))=(x \rightarrow \nabla x) \wedge(x \rightarrow(\nabla x \rightarrow x))=1$ and $(\nabla x \wedge(\nabla x \rightarrow x)) \rightarrow x=1$. Besides, (I23), (I6) and (I1) allow us to infer that $\nabla x \rightarrow \nabla(\nabla x \wedge(\nabla x \rightarrow x) \wedge x)=\nabla x \rightarrow \nabla(x \rightarrow(\nabla x \rightarrow x))=1$. In addition, from (I8), (I23), (I6) and (I26) we have $\nabla(\nabla x \wedge(\nabla x \rightarrow x)) \rightarrow \nabla(\nabla x \wedge(\nabla x \rightarrow x) \wedge x)=(\nabla x \wedge \nabla(\nabla x \rightarrow x)) \rightarrow \nabla x=1$. By these assertions and (I26) we conclude the proof.

(I39): From (I4), (I5) and (I31) we get that

$$
\begin{aligned}
\sim y \rightarrow \sim x & =(y \rightarrow 0) \rightarrow((\nabla y \rightarrow y) \rightarrow((x \rightarrow 0) \wedge(\nabla x \rightarrow x))) \\
& =(y \rightarrow 0) \rightarrow(((\nabla y \rightarrow y) \rightarrow(x \rightarrow 0)) \wedge((\nabla y \rightarrow y) \rightarrow(\nabla x \rightarrow x))) \\
& =(y \rightarrow 0) \rightarrow(((\nabla y \rightarrow y) \rightarrow(x \rightarrow 0)) \wedge(\nabla x \rightarrow(y \rightarrow x))) \\
& =((y \rightarrow 0) \rightarrow((\nabla y \rightarrow y) \rightarrow(x \rightarrow 0))) \wedge((y \rightarrow 0) \rightarrow(\nabla x \rightarrow(y \rightarrow x))) .
\end{aligned} .
$$

Then, by (I21), (I36), (I14) and (I2) we conclude that $\sim y \rightarrow \sim x=(\nabla x \rightarrow(y \rightarrow(0 \rightarrow x)))=1$. On the other hand, from (I35), (I38), (I19) and (I36) we have that

$\nabla \sim y \rightarrow \nabla(\sim y \wedge \sim x)=(y \rightarrow 0) \rightarrow((y \rightarrow 0) \wedge(x \rightarrow 0))=(y \rightarrow 0) \rightarrow(x \rightarrow 0)=1$. Hence, the above assertions and (I26) allow us to conclude that $\sim y \leq \sim x$.

Proposition 2.2. $\Theta(\mathcal{A})$ is a four-valued modal algebra.

Proof. We only have to prove that $\Theta(\mathcal{A})$ satisfies conditions (L1)-(L4).

(L1): Taking into account (I34), (I33), (I8) and (I15) we infer that

$$
\sim x=\nabla x \wedge((x \rightarrow 0) \rightarrow((x \rightarrow 0) \wedge(\nabla x \rightarrow x))) .
$$

then, by (I19), (I21), (I3) and (I30) we have that

$$
\sim \sim x=\nabla x \wedge((x \rightarrow 0) \wedge(\nabla x \rightarrow x))=\nabla x \wedge(\nabla x \rightarrow((x \rightarrow 0) \rightarrow x))=\nabla x \wedge(\nabla x \rightarrow x)=x .
$$


(L2): It is a direct consequence of (I39) and (L1).

(L3): From (I23) and (I10) we conclude that $(x \rightarrow 0) \vee \nabla x=1$. This identity and (I10) allow us to infer that $\sim x \vee \nabla x=1$.

(L4): It follows from (I6) and (I30).

Proposition 2.3. $\Theta$ is a full and faithful functor between $\mathrm{mdl}_{i}$-algebras and four-valued modal algebras.

Proof. It follows from Proposition 2.2 and the definition of $\Theta$. $\square$

Proposition 2.4. Every four-valued modal algebra $\mathcal{A}=\langle A, \wedge, \vee, \sim, \nabla, 0,1\rangle$ is isomorphic (equal) to $\Theta(\mathcal{B})$ for some $\mathrm{mdl}_{i}$-algebra $\mathcal{B}$.

Proof. Let $\mathcal{B}=\langle A, \wedge, \vee, \rightarrow, \nabla, 0,1\rangle$, where $x \rightarrow y=\nabla \sim x \vee y$ for all $x, y \in A$. As properties (I1)-(I12) hold in $\mathbb{M}_{4}$, then $\mathcal{B}$ is an $m d_{i}$-algebra. In order to complete the proof, we must show that $\Theta(\mathcal{B})=\mathcal{A}$. If we define $-x=(x \rightarrow 0) \wedge(\nabla x \rightarrow x)$, by Proposition 2.2 it follows that $\Theta(\mathcal{B})=\langle A, \wedge, \vee,-, \nabla, 0,1\rangle$ is a four-valued modal algebra. In addition, for every $x \in A, \sim x=(x \rightarrow 0) \wedge(\nabla x \rightarrow x)$, since this identity holds in $\mathbb{M}_{4} \cdot \square$

Theorem 2.1. $\Theta$ is a categorical equivalence between $\mathfrak{M D D} \mathfrak{L}_{i}$ and $\mathfrak{T} \mathfrak{M}$.

Proof. It is a direct consequence of Propositions 2.3, 2.4 and Theorem 1 of (MacLane, 1971). $\square$

Theorem 2.1 and the results on Section 1 confirm that $\mathrm{mdl}_{i}$-algebras are the algebraic counterpart of the four-valued Monteiro propositional calculus.

\section{References}

Bianco, E. (2004). Four-Valued Monteiro Propositional Calculus. XII Latin American Symposium on Mathematical Logic. Abstracts of Contributed Papers, San José, 3.

Bianco, E. (2008). Una contribución al estudio de las álgebras de De Morgan modales 4-valuadas. Ms. Thesis, Baha Blanca: Universidad Nacional del Sur.

Birkhoff, G. (1967). Lattice Theory (3rd ed.). Providence: American Mathematical Society, Col Pub.

Blackburn, P., de Rijke, M., \& Venema, Y. (2001). Modal Logic. Cambridge: Cambridge University Press. http://dx.doi.org/10.1017/CBO9781107050884

Burris, S., \& Sankappanavar, H. P. (1981). A Course in Universal Algebra. Graduate Texts in Mathematics 78. Berlin: Springer. http://dx.doi.org/10.1007/978-1-4613-8130-3

Carnielli, W. A., \& Marcos, J. (2002). A Taxonomy of C-Systems. In W. A. Carnielli, M. E. Coniglio, \& I. M. L. D’ottaviano (Eds.), Paraconsistency-The Logical Way to the Inconsistent, Volume 228 of Lecture Notes in Pure and Applied Mathematics (pp. 1-94). New York: Marcel Dekker.

Coniglio, M. E., \& Figallo, M. (2013). Hilbert-Style Presentations of Two Logics Associated to Tetravalent Modal Algebras. Studia Logica.

Figallo, A., \& Landini, P. (1995). On Generalized I-Algebras and Modal 4-Valued Algebras. Rep Math Logic Impact Factor, 29, 3-18.

Figallo, A., \& Ziliani, A. (1991). Symmetric Tetra-Valued Modal Algebras. Discreta Notas de la Sociedad de Matem tica de Chile, 10, 133-141.

Figallo, A. V. (1992). On the Congruences in Four-Valued Modal Algebras. Portugaliae Mathematica, 49, $249-261$.

Figallo, A. V. (1994). Tópicos sobre álgebras modales 4-valuadas. Proceedings of the 9th Latin American Symposium on Mathematical Logic, 38, 145-157.

Font, J. M., \& Rius, M. (1990). A Four-Valued Modal Logic Arising from Monteiros's Last Algebras. Proceedings of the 20th International Symposium on Multiple-Valued Logic, IEEE Computer Society Press, 85-92.

Font, J. M., \& Rius, M. (2000). An Abstract Algebraic Logic Approach to Tetravalent Modal Logics. The Journal of Symbolic Logic, 65, 481-518. http://dx.doi.org/10.2307/2586552

Kalman, J. A. (1958). Lattices with Involution. Transactions of the American Mathematical Society, 87, 485-491. http://dx.doi.org/10.1090/S0002-9947-1958-0095135-X

Lemmon, E. J., \& Scott, D. (1977). An Introduction to Modal Logic. In K. Segerberg (Ed.), The Lemmon Notes (Vol. 11). American Philosophical Quarterly Monograph Series. Oxford: Basil Blackwell.

Loureiro, I. (1980). Homomorphism Kernels of a Tetravalent Modal Algebra. Portugaliae Mathematica, 39, $371-379$.

Loureiro, I. (1982). Axiomatisation et propriétés des algèbres modales tétravalentes. Comptes Rendus de l'Académie des Sciences, 295, 555-557.

Loureiro, I. (1983a). Algebras Modais Tetravalentes. Ph. D. Thesis, Lisbon: Faculdade de Ciências de Lisboa.

Loureiro, I. (1983b). Prime Spectrum of a Tetravalent Modal Algebras. Notre Dame Journal of Formal Logic, $24,389-394$. 
http://dx.doi.org/10.1305/ndjfl/1093870382

Loureiro, I. (1983c). Finitely Generated Free Tetravalent Modal Algebras. Discrete Mathematics, 46, 41-48. http://dx.doi.org/10.1016/0012-365X(83)90268-6

Loureiro, I. (1984). Finite Tetravalent Modal Algebras. Revista de la Unión Matemática Argentina, 31, $187-191$.

Loureiro, I. (1985). Principal Congruences of Tetravalent Modal Algebras. Notre Dame Journal of Formal Logic, 26 , 76-80. http://dx.doi.org/10.1305/ndjfl/1093870762

MacLane, S. (1971). Categories for the Working Mathematician. Berlin: Springer.

Marona, R. (1964). A Characterisation of Morgan Lattices. Notas de Lógica Matemática, 18, 1-3.

Monteiro, A. (1960). Matrices de Morgan caractéristiques pour le calcul propositionnel classique. Anais da Academia Brasileira de Ciências, 52, 1-7.

Rasiowa, H. (1974). An Algebraic Approach to Non-Classical Logics. Warzawa \& Amsterdam: North-Holland Publishing Company.

Scroggs, S. J. (1951). Extensions of the Lewis System S5. The Journal of Symbolic Logic, 16, 112-120. http://dx.doi.org/10.2307/2266683 
Scientific Research Publishing (SCIRP) is one of the largest Open Access journal publishers. It is currently publishing more than 200 open access, online, peer-reviewed journals covering a wide range of academic disciplines. SCIRP serves the worldwide academic communities and contributes to the progress and application of science with its publication.

Other selected journals from SCIRP are listed as below. Submit your manuscript to us via either submit@scirp.org or Online Submission Portal.
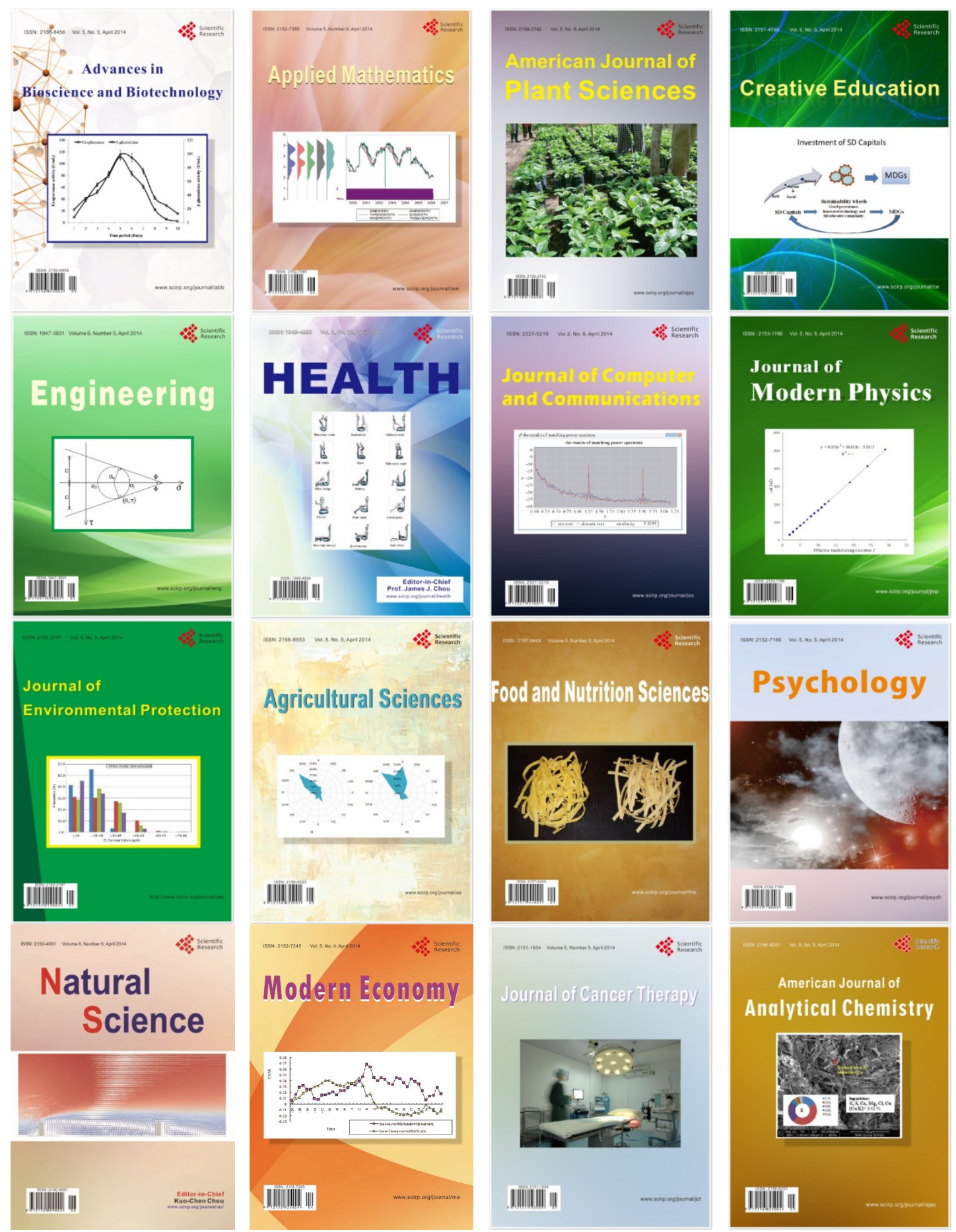